Conclusion: Plasma proteome analysis has identified a protein signature associated with response to RTX in SLE. These findings will be validated in independent cohorts and may offer the ability to predict response to RTX in lupus patients.

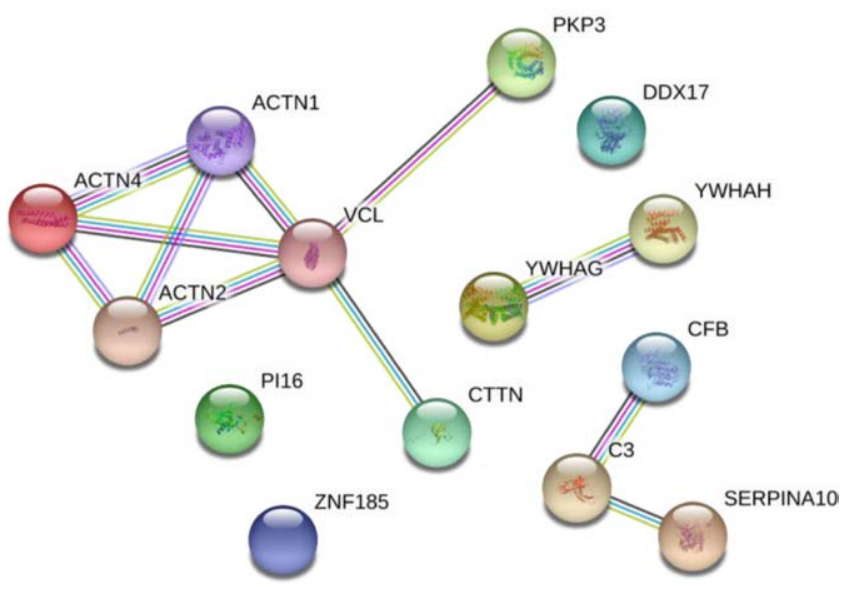

Protein Interaction Network of the 9 proteins from the multivariable model (with one additional level added). Cyan/magenta lines shown known interactions. Other colours show predicted interactions.

Disclosure of Interests: : John Reynolds: None declared, Jennifer Prattley: None declared, Mark Lunt: None declared, Ian N. Bruce Grant/research support from: Genzyme Sanofi, GSK, and UCB, Consultant of: Eli Lilly, AstraZeneca, UCB, Iltoo, and Merck Serono, Speakers bureau: UCB DOI: 10.1136/annrheumdis-2020-eular.3227

\section{SAT0192 \\ TRAJECTORIES OF LUPUS DISEASE ACTIVITY OVER TIME IN THE CLINICAL TRIAL SETTING REVEALED BY LATENT CLASS MIXED MODELS}

J. Reynolds ${ }^{1}, 2$, J. Prattley ${ }^{3}$, M. Lunt ${ }^{3}$, N. Geifman ${ }^{4}$, I. N. Bruce ${ }^{3,5} .{ }^{1}$ University of Birmingham, Institute of Inflammation and Ageing, Birmingham, United Kingdom; ' ${ }^{2}$ Sandwell and West Birmingham Hospitals NHS Trust, Rheumatology Department, Birmingham, United Kingdom; ${ }^{3}$ University of Manchester, Division of Musculoskeletal and Dermatological Sciences, Manchester, United Kingdom; ${ }^{4}$ University of Manchester, Division of Informatics, Imaging \& Data Sciences, Manchester, United Kingdom; ${ }^{5}$ Manchester University NHS Foundation Trust, NIHR Biomedical Research Centre, Manchester, United Kingdom

Background: There is considerable debate regarding the most appropriate outcome measures for clinical trials in systemic lupus erythematosus (SLE). We propose that studying changes in disease activity over time may yield important information above and beyond fixed endpoints at 12 months.

Objectives: To compare outcomes and predictors of response using i) traditional response definitions at a fixed time point and ii) trajectories of disease activity over time in a clinical trial dataset.

Methods: Data from the phase 3 clinical trial of Epratuzumab (EMBODY) in patients with active SLE were reanalysed. Response at 12 months was defined as: reduction to $\leq 1$ BILAG $B$ score, no increase in SLEDAI-2K score and no increase in prednisolone dose. Latent class mixed models (LCMM) were constructed using the numerical BILAG-2004 (nBILAG) score (where $A=12, B=8$, $\mathrm{C}=1, \mathrm{D} / \mathrm{E}=0$ ) from the screening visit until trial end. Cumulative prednisolone and Epratuzumab exposure was determined by estimating the area under the dosetime or concentration-time curves (AUC) respectively.

Results: 1202 patients were included with a median disease duration of 5.6 $[1.9,12.7]$ years and baseline nBILAG score of $20[16,24]$. At 12 months there were $564 / 1202(46.9 \%)$ responders. There was no association between receiving Epratuzumab and response. Responders were older, with lower baseline nBILAG score and baseline steroid dose. Using a cubic spline LCMM in the whole cohort, 5 trajectories were identified: Non-responders (NR, 33.9\%), rapid responders (RR, 36.7\%), slow responders (SR, 20.7\%), flare ( $F, 4.7 \%$ ) and high disease activity (HDA, 3.9\%) (figure). Of the 56412 -month responders, $113(20.4 \%)$ were in the NR latent class whilst 417 (73.9\%) were in RR or $\mathrm{SR}$ classes. Whilst there were no differences in study drop-out due to adverse events, withdrawal due to inefficacy was $20.6 \%$ in NR compared to $8.4 \%$ in RR and $7.6 \%$ in SR.

In multivariable logistic regression models adjusted for age, gender and study drop-out, being a responder (RR or SR) was significantly associated with activity in constitutional, musculoskeletal and cardiorespiratory BILAG domains, higher baseline and cumulative steroid exposure, and receiving active drug.

There was a trend towards higher baseline CD19+ counts in RR compared to NR or $\mathrm{SR}(\mathrm{p}=0.108$ ) but in a population-averaged multivariable linear model only baseline CD19+ count, Epratuzumab and steroid dose were associated with number of CD19+ cell over time ( $p<0.001$ for each).

Conclusion: Compared to a fixed end-point at 12 months, LCMM identified trajectories of disease activity and an association between clinical improvement and receiving active drug. Predictors of response vary significantly according to the response definition used.

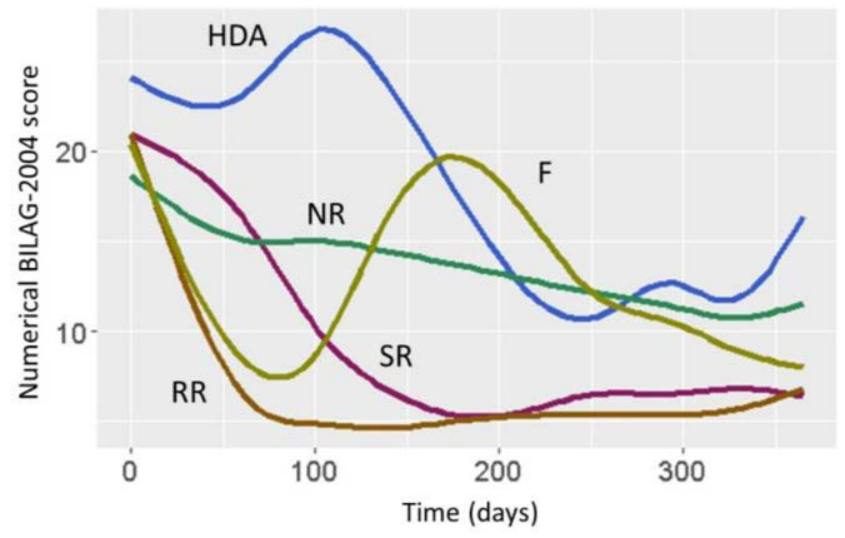

Table. Comparison of predictors of response between the 2 models

\begin{tabular}{lcc}
\hline & Response at 12 months & Response latent call (RR or SR) \\
\hline Age & Yes & No \\
Baseline gBILAG score & Yes (negative) & Yes (positive) \\
Baseline steroid dose & Yes & Yes \\
Active disease & & \\
Constitutional & No & Yes \\
Musculoskeletal & No & Yes \\
Cardiorespiratory & No & Yes \\
Gastroenterological & Yes & No \\
Anti-Smith & Yes & No \\
Low C3 & Yes & No \\
Epratuzumab & No & Yes \\
\end{tabular}

Acknowledgments: Presented on behalf of the MASTERPLANS Consortium Disclosure of Interests: John Reynolds: None declared, Jennifer Prattley: None declared, Mark Lunt: None declared, Nophar Geifman: None declared, lan N. Bruce Grant/research support from: Genzyme Sanofi, GSK, and UCB, Consultant of: Eli Lilly, AstraZeneca, UCB, lltoo, and Merck Serono, Speakers bureau: UCB DOI: 10.1136/annrheumdis-2020-eular.3611

\section{SAT0193 A PHASE 3, OPEN-LABEL, CONTINUATION STUDY EVALUATING LONG-TERM SAFETY AND EFFICACY OF BELIMUMAB IN PATIENTS FROM JAPAN AND KOREA WITH SYSTEMIC LUPUS ERYTHEMATOSUS, FOR UP TO 7 YEARS}

Y. Tanaka ${ }^{1}$, S. C. Bae ${ }^{2}$, D. Bass ${ }^{3}$, M. Chu ${ }^{4}$, P. Curtis ${ }^{5}$, K. Derose ${ }^{3}$, B. Ji ${ }^{5}$, R. Kurrasch ${ }^{3}$, J. Lowe ${ }^{5}$, P. Meizlik ${ }^{3}$, D. Roth ${ }^{3}{ }^{1}{ }^{\text {University of Occupational and }}$ Environmental Health, Kitakyushu, Japan; ${ }^{2}$ Hanyang University Hospital for Rheumatic Diseases, Seoul, Korea, Rep. of (South Korea); ${ }^{3}$ GlaxoSmithKline, Collegeville, United States of America; ${ }^{4}$ GlaxoSmithKline (at the time of the study), Collegeville, United States of America; ${ }^{5}$ GlaxoSmithKline, Uxbridge, United Kingdom

Background: Systemic lupus erythematosus (SLE) is an autoimmune disorder more prevalent in the Asian population vs Caucasians. Belimumab (BEL), a monoclonal antibody targeting B-lymphocyte stimulator, is approved in patients (pts) $\geq 5$ years with active, autoantibody-positive SLE.

Objectives: Evaluate long-term safety and efficacy of intravenous (IV) BEL + standard SLE therapy (SST) in pts with SLE in Japan/Korea.

Methods: In this Phase 3, multicentre, open-label (OL) study (BEL114333; NCT01597622), eligible ( $\geq 18$ years of age) completers of the double-blind phase of GSK study BEL113750 in Japan and South Korea or the subcutaneous OL phase of GSK Study BEL112341 in Japan, received monthly BEL $10 \mathrm{mg} / \mathrm{kg}$ IV 